\title{
DESIGN AND ANALYSIS OF A RECONFIGURABLE INTELLIGENT META-SURFACE FOR VEHICULAR NETWORKS
}

\author{
Mohammad Ojaroudi ${ }^{1}$, Valeria Loscrí ${ }^{1}$, Anna Maria Vegni ${ }^{2}$ \\ ${ }^{1}$ Inria Lille - Nord Europe, ${ }^{2}$ Roma Tre University, Rome, Italy \\ NOTE: Corresponding author: Valeria Loscrí, valeria.loscri@inria.fr
}

\begin{abstract}
In this work, a new paradigm for vehicular communications based on Reconfigurable Intelligent Meta-surfaces (RIMs) is presented. By using the proposed RIM, we are able to manipulate electromagnetic waves in the half-space, since the element is reflective. The unit cell consists of a U-shaped designed microstrip structure equipped with a pin diode and via a hole. In this study, two different reflection modes are achieved for 1-bit data transferring in each state. By incorporating these two different configurations together, the reflected phases in the proposed RIM surface can be controlled respectively in $0^{\circ}$ and $180^{\circ}$. The proposed unit cell can provide a usable double negative functional characteristic around $5.3 \mathrm{GHz}$. The main goal of this paper is the use of a multifunctional behavior RIM for vehicular communications to code the transmitted wave. A novel phase distribution diagram is generated to propagate in each angle. Moreover, two major electromagnetic modulation functions, beam forming and space coding have been demonstrated. Finally, we show how the RIM can be employed for vehicular communications, acting as a coated access point along the street. We derive the instantaneous data rate at the receiver node, the outage probability and the channel capacity, as affected by different beam widths, distances and vehicle speed.
\end{abstract}

Keywords - Beam width, Instantaneous data rate, Reconfigurable meta-surfaces, V2X communications

\section{INTRODUCTION}

In recent years, special attention has been paid to driverless cars using automotive radars [7, 8]. A Vehicle-toEverything (V2X) communication paradigm has emerged as one of the most important enabling technologies for vehicular networks. This has the potential of making streets and highways safer, the traffic more efficient and less harmful to the environment [9]. An example is the exchange of traffic information, as in the Self-Organizing Traffic Information System approach [12].

One of the most important challenges arising in the V2X paradigm is the high dynamic and the need of highly efficient communication solutions in order to "follow" the rapid changing of communication nodes. Recently, an innovative wireless communication paradigm based on the utilization of specific features of controllable metasurfaces has been investigated in $[2,3,4,5,6,15]$. With the advancement of technology in metamaterials, metasurfaces which are a thin two-dimensional structure of metamaterials [1], due to their unique properties like the capability to provide abrupt phase shift, amplitude modulation, and polarization conversion of the electromagnetic (EM) wave, have found a variety of applications in various fields of science, such as physics, engineering, biotechnology, and telecommunications [18].

Reconfigurable Intelligent Meta-surfaces (RIMs) can be very effective for improving the performance of a V2X communication system, especially when the Line-of-Sight of the propagating signal is not available. [22] and [23] are among the first contributions of exploitation of RIM in vehicular communications. In [22], the authors focus on the physical layer security based on the exploitation of a RIM or RIS (Reconfigurable Intelligent Surface). They derive the average secrecy capacity of the system, by considering an ideal reconfigurable meta-surface. They do not give any detail about the design of a real meta-surface with the needed characteristics in order to obtain the specific behavior as described in the paper. Also in [23], the authors show the rich potentiality of the RIM integration in a vehicular communication system, by analysing a hypothetic ideal controllable meta-surface. Anyway, no detail about the features of a real meta-surface is provided in the paper. In [17], we have proposed a unit cell configuration working at millimiter-waves in order to control the phase shift from 0 to $180^{\circ}$. Specifically, we have investigated the unit cell design without evaluating the full structure and relate the capability to control the beam width based on the number of the unit cells of the full structure. Indeed, the higher is the number of the unit cells of the full structure, the better is the capacity to control the beam width of the radiation pattern generated as the reflected wave of an impinging signal.

As demonstrated in [11], it is possible to accurately model the number of cells as sources of secondary radiation [16] by applying the Huygens principle in the far-field limit. In practice, the higher is the number of unit cells we consider in the full structure, the better is the capability to control the phase, but above all to control the beam width of the 
main beam for beam stearing objective.

In [18], it is demonstrated how this type of meta-surface allows a digital control of EM waves, by associating two coding elements with opposite reflection phases (i.e., $0^{\circ}$ and $180^{\circ}$ ) and considering them as digital bits (i.e., 0 and 1 in the binary case). Reconfigurable meta-surface structures can be applied to manipulate EM waves in a simple and effective way, by changing the coding elements on a 2D plane with predesigned coding sequences [19]. In order to independently control and create different coding sequences a Field Programmable Gate Array (FPGA) is used. By changing the coding sequences stored in the FPGA, many different functionalities can be switched in real time, thereby leading to programmable metasurfaces.

In this paper, we formulate the specific features that a meta-surface for vehicular communication applications needs to have in the frequency range of $[5,5.9] \mathrm{GHz}$. In particular, we consider a tracking application with beam steering, for which is of paramount importance to control the phase and to concentrate the power in the main lobe of the reflected signal, as much as possible. In order to meet these specific requirements, we will design a unit cell and then derive a full structure constituted by the periodic repetitions of these unit cells, behaving as a reflector for a pair of transmitter receivers. We will focus on the phase shift and the main beam width in order to design a beam tracking system which is able to "follow" the mobile receiver node for improving the efficiency in terms of data rate and outage probability. Based on that, we will present a multifunctional reconfigurable metasurface structure based on the radiation pattern modulation of the reflection coefficient. Firstly, we will design a reconfigurable U-shaped unit cell using a pin diode via a hole, which can provide $180^{\circ}$-phase difference between $\mathrm{ON}$ and OFF states. Specifically, a $10 \times 10$ meta-surface loaded with PIN diodes is designed for multifunctional behavior, such as coding and beam steering. The simulated results in both scenarios of unit cell and full structures will show the effectiveness of the proposed structure for vehicular communications.

Our main contributions can be summarized as follows:

- We design a specific meta-atom working at $5.3 \mathrm{GHz}$ for automotive applications and based on this unit cell, we design a full structure and validate it to assess its suitability for the vehicular application considered;

- We design a full structure and validate it to assess its suitability for the vehicular application considered;

- We consider the integration of the designed Reconfigurable Intelligent Meta-surface (RIM) in a vehicular system and we numerically evaluate the performance; We compare the results of the system with and without RIM in terms of outage probability and capacity, by demonstrating the great potentiality of this type of structure.

The rest of the article is organized as follows. Section 2 describes the specific scenario considered and details the proposed unit cell structure. Section 3 presents simulated results for the proposed RIM unit cell in two ON and OFF states, expressed in terms of $(i)$ reflection magnitude and phase, and (ii) effective permittivity and permeability. In Section 4 we validate the full RIM structure, while in Section 5 the performance of the proposed RIM has been exploited for vehicular communications, by considering that the RSU and the receiver vehicle are coated with the implemented $10 \times 10$ unit cells RIM. Instantaneous data rate, outage probability and channel capacity have been obtained as validation results. In Section 6, we present a few considerations related to the obtained results. Finally, conclusions are drawn at the end of the article.

\section{UNIT-CELL DESIGN AND CONFIGURA- TION}

The envisioned vehicular communications paradigm based on using RIM is shown in Fig. 1. In particular, we focus on a RIM relay based scheme. An RSU (Road Side Unit) is at the side of the road and is coated with the specific meta-surface structure we will detail later. We assume that the RSU, as represented in Fig. 1, is re-transmitting data to the receiver (i.e., the green vehicle). In order to maximize the SNR to the destination, the RIM RSU will be "beam tracking" the receiver. This specific behavior can be realized by designing a unit cell with specific features as explained to the follow.

The proposed unit cell structure is shown in Fig. $2(a)$. In this structure we proposed a U-shaped microstrip structure which is able to be reconfigurable by a PIN diode. By creating a rectangular slot in the corner of the rectangular patch a new path of surface current will be created. Hence, we can control the input impedance of the unit cell which is suitable for such an application of RIM. Moreover, the relevant equivalent circuit for this structure is shown in Fig. $2(b)$. Regarding the ON and OFF states of the PIN diode, we put two different equivalent circuits for this section. All the dimensions are summarized in Table 1.

Table 1 - Parameters of the proposed unit cell

\begin{tabular}{c|c|c|c}
\hline \hline Parameter & Value [mm] & Parameter & Value [mm] \\
\hline$W_{S u b}$ & 10 & $L_{C}$ & 9 \\
\hline$L_{S u b}$ & 10 & $W_{C}$ & 6 \\
\hline$h_{S u b}$ & 1.6 & $W_{C 1}$ & 2 \\
\hline$L_{d}$ & 1 & $L_{C 1}$ & 3 \\
\hline$W_{d}$ & 3 & $R_{v i a}$ & 0.25 \\
\hline \hline
\end{tabular}

In meta-surface structures, beam steering can be consid- 


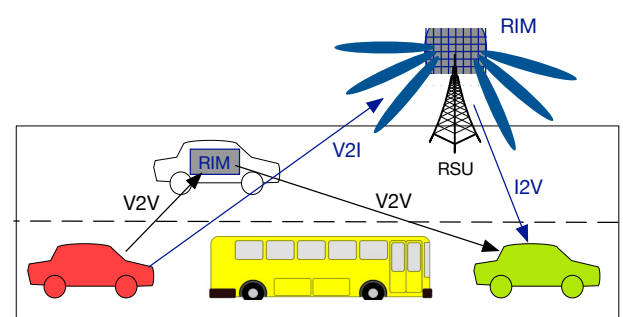

Fig. 1 - Vehicular communications paradigm based on the use of RIMs as relay node, for data transmission to a receiver node (green vehicle), in case of Vehicle-to-Vehicle (black lines) and Vehicle-toInfrastructure/Infrastructure-to-Vehicle (blue lines)

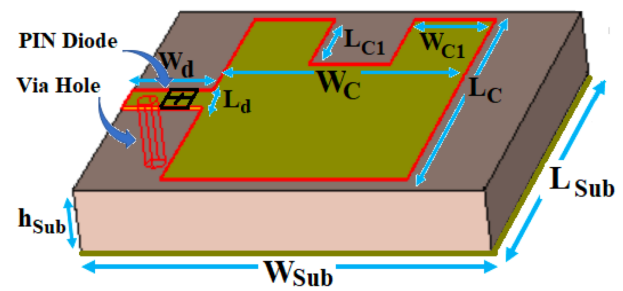

(a)

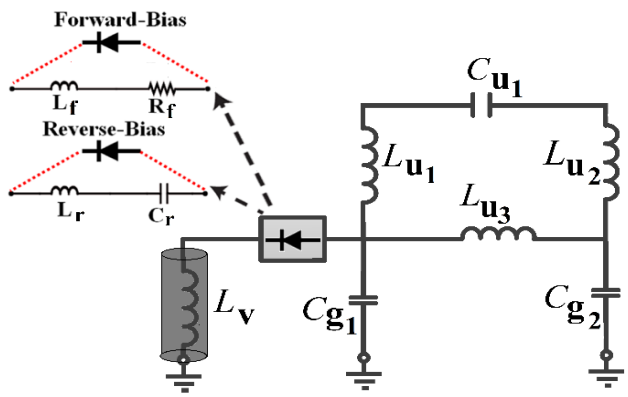

(b)

Fig. 2 - $(a)$ The configuration and the geometry of the proposed unit cell with a U-shaped radiating patch, and (b) unit cells with PIN diode's equivalent circuit model for ON and OFF states.

ered as a particular case of wavefront manipulation that occurs in the far field. Regarding the Huygens principle, the meta-surface structures can be considered as an integrated EM radiator array [20]. Herein, in order to model the meta-antenna array, we consider a method that has been validated in several works via extensive simulations [21]. Considering each unit cell as an element of the planar array, the far field of the meta-surface can be obtained as:

$$
F(\theta, \Phi)=f_{E}(\theta, \Phi) \cdot f_{A}(\theta, \Phi)
$$

by considering infinite sphere, $\theta$ is the elevation angle, $\Phi$ is the azimuth angle of an arbitrary direction in this coordination.

Regarding the planar array, the pattern function of each unit cell $f_{E}(\theta, \Phi)$ is the element factor and the pattern function of full planar configuration $f_{A}(\theta, \Phi)$ is the array factor. In far field region, we assume a planar wave covers the entire meta-surface. Therefore, the radiated pattern will depend only on the array factor. In this case, the far field pattern for the meta-surface with $N \times M$ unit cells, becomes

$$
\begin{array}{r}
F(\theta, \Phi)=\sum_{m=1}^{M} \sum_{n=1}^{N} A_{m n} e^{j / \alpha_{m n}} f_{m n}\left(\theta_{i}, \Phi_{i}\right) . \\
\Gamma_{m n} e^{j / \Phi_{m n}} f_{m n}(\theta, \Phi) e^{j k_{0 m n}(\theta, \Phi)},
\end{array}
$$

where $A_{m n}$ and $\alpha_{m n}$ are the amplitude and phase of the wave incident to the $(m, n)$-th unit cell, respectively, with $m=[1,2, \ldots, M]$ and $n=[1,2, \ldots, N]$. In Eq. (2), $\Gamma_{m n}$ and $\Phi_{m n}$ are the amplitude and phase of the response of the $(m, n)$-th unit cell, respectively; $f_{m n}(\theta, \phi)$ denotes the scattering diagram of the $(m, n)$-th unit cell towards an arbitrary direction of reflection, whereas $f_{m n}\left(\theta_{i}, \Phi_{i}\right)$ denotes the response of the $(m, n)$-th unit cell at the direction of incidence determined by $\theta_{i}, \Phi_{i}$ and $k_{0}=2 \pi / \lambda_{0}$ is the wave number. Finally, we introduce $\zeta_{m n}(\theta, \Phi)$, which denotes the relative phase shift of the unit cells with respect to the radiation pattern coordinates, given by

$$
\zeta_{m n}(\theta, \Phi)=D_{u} \sin (\theta)\left[\left(m-\frac{1}{2}\right) \cos (\Phi)+\left(n-\frac{1}{2}\right) \sin (\Phi)\right],
$$

with $D_{u}[\mathrm{~m}]$ as the unit cell size.

In order to make the model able to be calculated, we make a further assumption in the point of plane incident wave view, so that factors $A_{m n}, \alpha_{m n}$, and $f_{m n}\left(\theta_{i}, \Phi_{i}\right)$ are constants for all $m$ and $n$ indexes. In addition, we apply the widespread assumption to the scattering pattern of the unit cell, which is modeled over the positive semisphere with the function $\cos (\theta)$, which is a widespread assumption, [11]. Finally, and without loss of generality, we consider the normal incidence i.e., $\left(\theta_{i}=\Phi_{i}=0\right)$. Then, Eq. (2) becomes [11]

$$
E(\theta, \Phi)=k \cos (\theta) \sum_{m=1}^{M} \sum_{n=1}^{N} \Gamma_{m n} e^{j}\left[\Phi_{m n}+k_{0} \zeta_{m n}(\theta, \Phi)\right]
$$

with $k$ as a constant.

In order to have anomalous reflection, the main objective is controlling the phase shift of the unit cells $\Phi_{m n}$. In particular, we manipulate the phase of the reflected waveform but not its amplitude. In this current version we do not focus on the control scheme for our system, since it is out of scope for this work. In reconfigurable meta-surface generating different coding sequence for unit cells, we are able to achieve desired functionalities such as beam steering and radiated wave modulation. In this regard, the amplitude $\Gamma_{m n}$ and phase $\Phi_{m n}$ of the $(m, n)$-th unit cell need to be determined somehow which the entire response of the array matches with the required functionality. After this step, by mapping the required $\Gamma$ and $\Phi$ to the closest available unit cell states, the desired functionality will be obtained. In the case of anomalous reflection for beam steering, analytical methods provide high accuracy.

In this study, in order to obtain beam steering functionality, the phase gradient approach is used to determine the direction of reflection [13]. Considering $\Phi(x, y)$ as the phase profile which is imposed by reconfigurable metasurface, the virtual wave vector $\mathbf{K}_{\Phi}=\Phi_{x} \hat{\mathbf{x}}+\Phi_{y} \hat{\mathbf{y}}$ can be 
assigned to the meta-surface. In this context, the momentum conservation law for wave vectors can be expressed as

$$
\begin{gathered}
k_{i} \sin \left(\theta_{i}\right) \cos \left(\Phi_{i}\right)+\frac{\partial \Phi_{x}}{\partial x}=k_{r} \sin \left(\theta_{r}\right) \cos \left(\Phi_{r}\right) \\
k_{i} \sin \left(\theta_{i}\right) \cos \left(\Phi_{i}\right)+\frac{\partial \Phi_{y}}{\partial y}=k_{r} \sin \left(\theta_{r}\right) \sin \left(\Phi_{r}\right)
\end{gathered}
$$

where $\partial \Phi_{x} / \partial x$ and $\partial \Phi_{y} / \partial y$ describe the gradients along the $\hat{\mathbf{x}}$ - and $\hat{\mathbf{y}}$ - directions, respectively. For simplicity we consider the normal incident wave case i.e., $\left(\theta_{i}=\Phi_{i}=0\right)$ in lossless medium scenario [14]. Assuming air as the medium of the incident and reflected wave, we can simplify the formulations above as

$$
\partial \Phi_{x}=\frac{2 \pi \partial x \cos \Phi_{r} \sin \theta_{r}}{\lambda_{0}}, \partial \Phi_{y}=\frac{2 \pi \partial y \sin \Phi_{r} \sin \theta_{r}}{\lambda_{0}},
$$

which demonstrate the phase shift $\Phi_{x}$ and $\Phi_{y}$ that need to be performed per unit of distance (i.e., $\partial x$ and $\partial y$ ) along the $\hat{\mathbf{x}}$ and $\hat{\mathbf{y}}$ directions, respectively. Then, in Eq. (6) we set the unit cell size as $\partial x=\partial y=D_{u}$, in order to obtain the phase required at the $(m, n)$-th unit cell as

$$
\Phi_{m n}=\frac{2 \pi D_{u}\left(m \cos \Phi_{r} \sin \theta_{r}+n \sin \Phi_{r} \sin \theta_{r}\right)}{\lambda_{0}} .
$$

For beam-steering functionality the required phase $\Phi_{m n}$ is calculated for all the unit cells, to assign radiated states to each unit cell. Then, a closest neighbor mapping is done between the required phase and that provided by the different unit cell states.

\section{SIMULATION RESULTS}

In this section, we evaluate the performance of the unit cell we designed. The simulation is realized by the means of a commercial software CST studio suite. The specific configuration considered in CST is the boundary condition as unit cell in $\hat{\mathbf{x}}$ - and $\hat{\mathbf{y}}$-directions with open-add space in $\hat{\mathbf{z}}$-direction. The reflectivity and reflection phases of unit cells are simulated using the frequency domain solver. The simulated reflection magnitude and phase of the unit cell are shown in Fig. 3. It is obvious that at 5.3 $\mathrm{GHz}$, while the reflection magnitude is almost identical between the ON and OFF state, the reflection phase between the two cases has a $180^{\circ}$ change. The maximum unit cell loss is around $2.5 \mathrm{~dB}$ for the OFF state. It is evident that the proposed 1-bit unit cell is suitable for the multifunctional meta-surface such as coding and beam steering. As the phase change between ON/OFF states is relative, we can simply state that a unit cell with an $\mathrm{ON}$ state corresponds to a $0^{\circ}$ phase reflection, while one with an $\mathrm{OFF}$ state has $\mathrm{a}-180^{\circ}$ phase reflection.

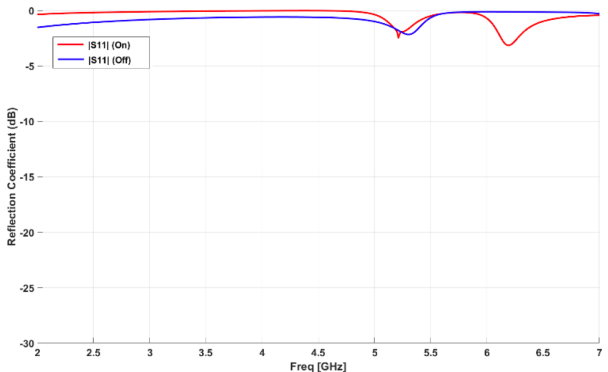

(a)

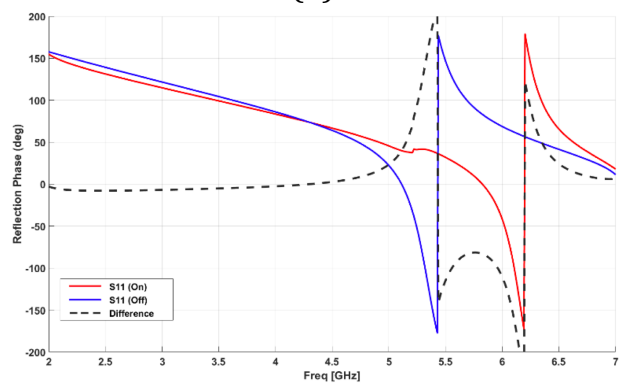

(b)

Fig. 3 - Simulated results for the proposed unit cell in two ON and OFF situations, expressed in terms of $(a)$ reflection magnitude and $(b)$ reflection phases.

In order to assess the double negative characteristics, $S_{11}$ and $S_{21}$ reflection and transmission coefficients are extracted from the design in CST in both magnitude and angle (expressed in rad). Then, the effective permittivity $\varepsilon_{\text {eff }}$ and effective permeability $\mu_{\text {eff }}$ are obtained respectively as [20]:

$$
\varepsilon_{e f f}=\frac{\frac{1}{k d} \cos ^{-1}\left[\frac{1}{2 S_{21}}\left(1-S_{11}^{2}+S_{21}^{2}\right)\right]}{\sqrt{\frac{\left(1+S_{11}\right)^{2}-S_{21}^{2}}{\left(1-S_{11}\right)^{2}-S_{21}^{2}}}},
$$

and

$$
\mu_{e f f}=\frac{1}{k d} \cos ^{-1}\left[\frac{1}{2 S_{21}}\left(1-S_{11}^{2}+S_{21}^{2}\right)\right] \sqrt{\frac{\left(1+S_{11}\right)^{2}-S_{21}^{2}}{\left(1-S_{11}\right)^{2}-S_{21}^{2}}},
$$

where $k$ denotes the wave number of the incident wave and $d[\mathrm{~mm}]$ is the thickness of the unit cell. In this study, the meta-surface is printed on an FR-4 substrate with thickness of $1.6 \mathrm{~mm}$ and is designed to increase the directivity and bandwidth of the structure. Fig. 4 shows the effective permittivity and effective permeability related to the designed unit cell. It is clear that the proposed unit cell has double-negative material characteristics around $5.3 \mathrm{GHz}$ for both ON and OFF states.

\section{FULL STRUCTURE DESCRIPTION AND EVALUATION}

In this section, in order to evaluate the performance of the proposed unit cell for multifunctional applications, a 


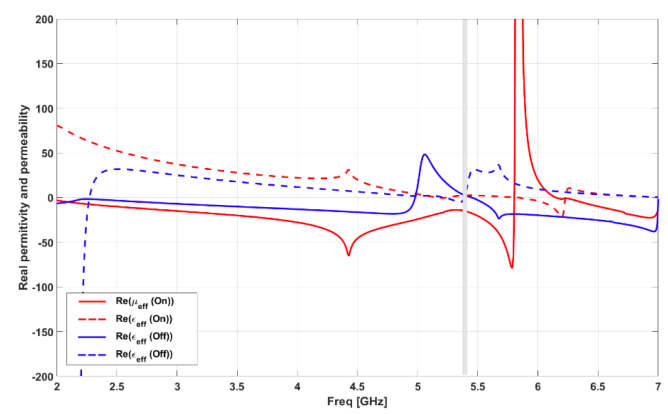

Fig. 4 - The effective permittivity and permeability of the unit cell.

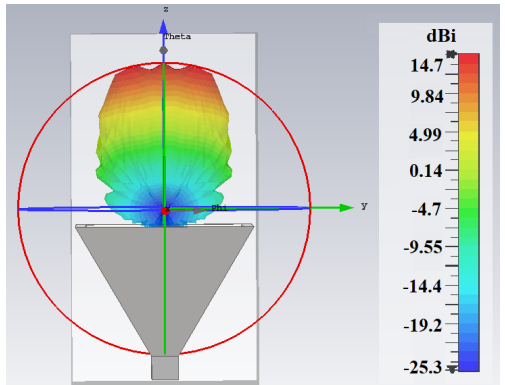

(a)

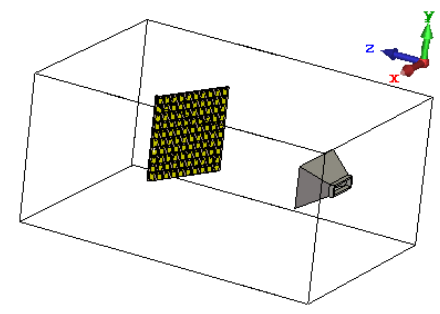

(b)

Fig. 5 - $(a)$ Horn antenna as a transmitter and $(b)$ the 1 -bit $10 \times 10$ meta-surface simulated model.

Horn antenna is employed as a main radiator at the transmitting side, as shown in Fig. $5(a)$. The redesigned Horn antenna is given to excite the meta-surface structure at $5.3 \mathrm{GHz}$. In addition, the 1 -bit $10 \times 10$ meta-surface simulated model is shown in Fig. $5(b)$.

\subsection{Coding Meta-surface Construction}

In this section we consider coding meta-surface based on the RIM structure, as the possibility to characterize the states $\mathrm{ON}$ and OFF as matching the bits 0 and 1. Fig. 6 (a) shows a random coding meta-surface with a fixed ratio and a different coding sequence, and Fig. $6(b)$ shows the simulated 3D radiation in full structure using CST. As shown clearly, with coding sequence, the diffusion of the far-field pattern and the scattering amplitude at the normal incident angle are apparently the same. According to the code MATLAB, a 3D far-field pattern can be obtained with fixed ratio between 0 and 1 coding elements. The simulated results are demonstrated as Fig. $6(c)$. Once ratio fixed, the scattering amplitude at the normal incident angle $0^{\circ}$ is determined, and the efficient of coding metasurface only depends on the uniformity of the scattering

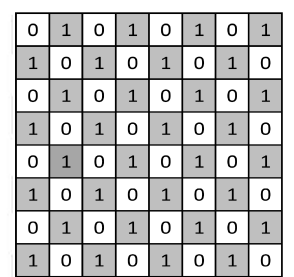

(a)

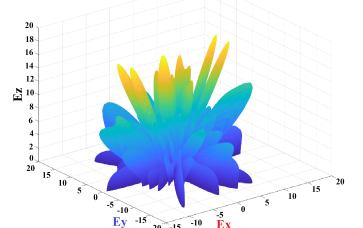

(c)

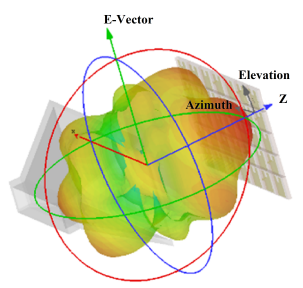

(b)

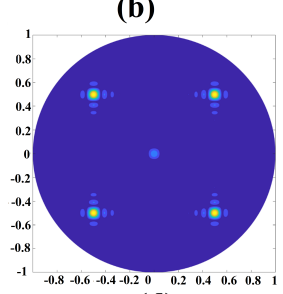

(d)
Fig. 6 - Reflection phase distribution and 3D pattern of the coding metasurface with Horn antenna as an EM source located at $(0 \mathrm{~cm}, 0 \mathrm{~cm}, 100$ $\mathrm{cm})$. (a) Ideal reflection phase distribution; $(b)$ 3D pattern in full structure; $(c)$ the beam pattern based on ideal phase distribution; $(d)$ the beam pattern contour for coding application.

beam. Fig. $6(d)$ demonstrates contour plot with corresponding 3D scattering pattern.

\subsection{Beam-Steering Meta-surface Construction}

In this section, an $8 \times 8$ beam steering meta-surface is modeled and simulated using CST Studio software to verify the beam steering capability of the RIM. In this simulation, the meta-surface is in the X-Y plane, and a horn serves as an EM source, which is located at $(50 \mathrm{~mm}, 0 \mathrm{~cm}$, $0 \mathrm{~cm})$ with a rotation of $\left(45^{\circ}, 0^{\circ}\right)$ with respect to the metasurface. Then, an ON/OFF pattern matrix for steering to $\left(120^{\circ}, 0^{\circ}\right)$ is loaded to the PIN diode of each unit cell. Finally, the simulation results are exported and shown in Fig. $7(a)$ and $(b)$. It is clearly observed that the coding meta-surface is capable of steering the beam to the desired direction with $20^{\circ}$ angular resolution in the full structure case.

\section{EVALUATION IN VEHICULAR APPLICA- TIONS}

The proposed RIM structure is now exploited for vehicular communications, as depicted in Fig. 1. Specifically, we consider the communication link in a vehicular context, established from a source (red vehicle) and a receiver node (green vehicle). We assume a highway scenario and the transmitter and receiver vehicles have constant speed for the transmission time window. In order to characterize the impact of the controllable meta-surface in the communication system, we assume a simplified system with no interferences caused by others vehicles. In order to quantify the impact of the RIM on the performance system, we will consider (i) the relation between different beam width on the average rate, given a certain estimated velocity, and (ii) the derivation of the outage probability and the analysis of the communication system in terms of 

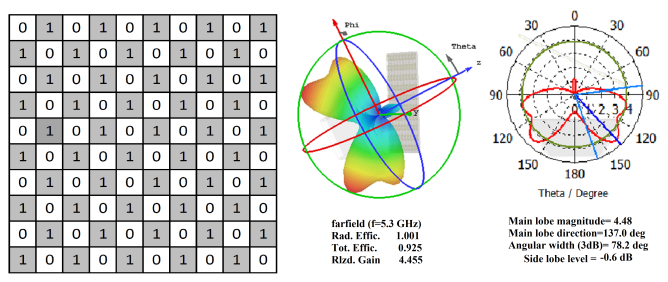

(a)
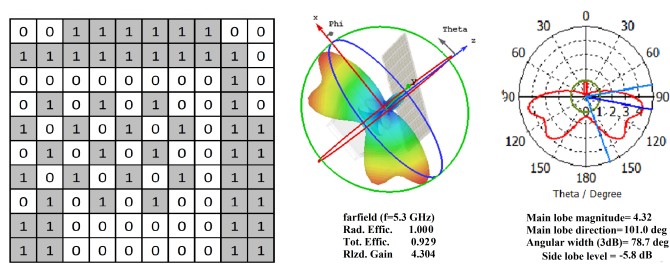

(b)

Fig. 7 - The simulation results of 3D gain total with the horn and metasurface and the $2 \mathrm{D}$ radiation pattern for $10 \times 10$ with $50 \mathrm{~mm}$ distance and $25^{\circ}$ rotation at $\mathrm{Y}$-plane at $5.25 \mathrm{GHz}$ with different phase distribution, $(a)$ uniform distribution and $(b)$ random distribution.

both outage and capacity, in cases with and without RIM.

\subsection{Beam width Impact Evaluation}

A $10 \times 10$ unit cells RIM is coating both the RSU (acting as relay node) and the receiver vehicle. In particular, we compute the instantaneous data rate by assuming a perfect alignment between the beam from the RSU and the receiver node. The instantaneous data rate is derived by the Shannon capacity formula for the instantaneous rate as:

$$
R\left(t, \theta_{b}\right)=B \log _{2}\left(1+S N R\left(t, \theta_{b}\right)\right),
$$

where $B[\mathrm{~Hz}]$ is the bandwidth of the system, $S N R$ [dB] is the Signal-to-Noise Ratio, $t[\mathrm{~s}]$ is the time instant, and $\theta_{b}$ is the beam width computed in degrees.

We assume that our system is able to "instantaneously" switch from the $(i-1)$-th beam to the $i$-th one, and the "transmitter" beam and the "receiver" one are perfectly aligned. Moreover, we consider a beam model where the gain inside the beam is uniform and zero outside. In Eq. (11), the expression of the $S N R$ can be written as

$$
\operatorname{SNR}\left(t, \theta_{b}\right)=\frac{P_{r x}\left(t, \theta_{b}\right)}{P_{\text {noise }}},
$$

where $P_{\text {noise }}[\mathrm{dB}]$ represents the thermal noise including a Noise Figure i.e., $N F[\mathrm{~dB}]$, which can be expressed as

$$
P_{\text {noise }}=-174+10 \log _{10} B+N F \text {. }
$$

In Eq. (12), the term $P_{r x}$ is the received power, expressed in linear scale as [10]:

$$
P_{r x}\left(t, \theta_{b}\right)=\mathcal{K}\left(\frac{\pi^{2}}{\theta_{b}^{2}}\right)^{2} \frac{1}{\left[(v t)^{2}+r^{2}\right]^{n / 2}},
$$

where $v[\mathrm{~m} / \mathrm{s}]$ is the vehicle speed, $n$ is the path loss exponent, and $r$ [m] is the distance from the RSU and the
Table 2 - Simulation Parameters

\begin{tabular}{c|c}
\hline \hline Parameter & Value \\
\hline$E I R P$ & $57 \mathrm{dBm}$ \\
\hline$B$ & $75 \mathrm{MHz}$ \\
\hline$f_{c}$ & $5.3 \mathrm{GHz}$ \\
\hline$r$ & {$[0,200] \mathrm{m}$} \\
\hline$N F$ & $6 \mathrm{~dB}$ \\
\hline$v$ & {$[25,50,75] \mathrm{km} / \mathrm{h}$} \\
\hline
\end{tabular}

receiver node. The term $\mathcal{K}$ is defined as

$$
10 \log _{10}(\mathcal{K})=E I R P_{d B m}-E+10 n \log _{10}(\lambda / 4),
$$

where EIRP is the Equivalent Isotropic Radiated Power and $\lambda[\mathrm{m}]$ is the wavelength and $E$ represents the shadowing margin. In order to compute $\mathcal{K}$, we consider the power associated with the reflected signal $S_{21}$ as computed in Eqs. (9) and (10).

Notice that we assume that the transmitter node is transmitting at an EIRP that for Europe is equal to $57 \mathrm{dBm}$. The relay will not be able to retransmit at full power since a percentage of the power associated to the impinging wave will be dissipated as a reflected signal. In particular, the design of the unit cell has been optimized in order to minimize this power loss i.e., a $2 \%$ of the total transmitted power will be wasted as a reflected component of the wave. Furthermore, without loss of generality, the speed of the receiver vehicle is considered constant during the transmission time, since the interval time of reception is short with respect to the variation of the vehicle speed. Table 2 collects the parameters used in the numerical results.

In Fig. $8(a)$ we show the instantaneous and ideal rate, i.e., the maximum achievable rate with the assumption of a perfect alignment and without error estimation of the velocity at the receiver by considering a narrow beam width, i.e., $\theta_{b}=[0.1,10]^{\circ}$. Data rate values range from $\approx[15,25]$ Gbps, where higher values are obtained for smaller beam widths, while a data rate decrease is observed for larger beam widths.

Furthermore, we have considered the impact of different speeds on the achievable rate and we can observe that, as expected, the data rate decreases for increasing vehicle speed. Finally, the effect of the distance on the data rate is a decrease of performance when the distance increases.

In Fig. $8(b)$ we show the impact of a wider beam width for the same scenario. Of course, wider beam widths are related with a smaller number of unit cells. Indeed, the smaller is the number of unit cells, the lower is the fine control we can realize on the RIM. As expected, we observe a decreasing of the instantaneous data rate from a maximum of $23 \mathrm{Gbps}$ in Fig. $8(b)$ to a maximum of $15 \mathrm{Gbps}$ in Fig. $8(a)$. 


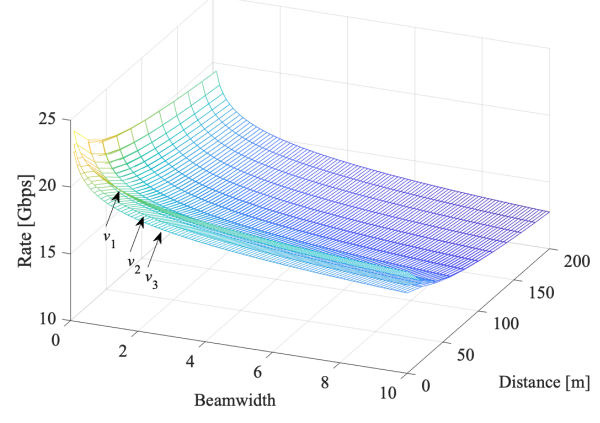

(a)

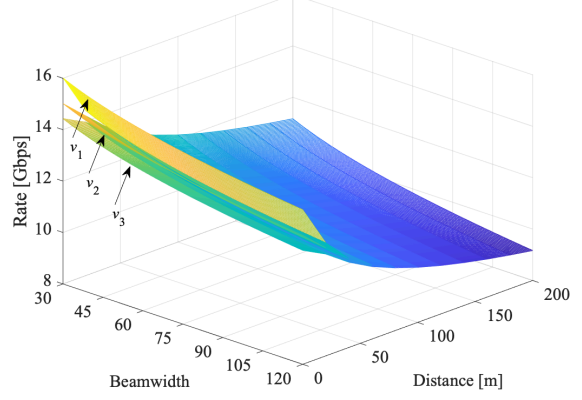

(b)

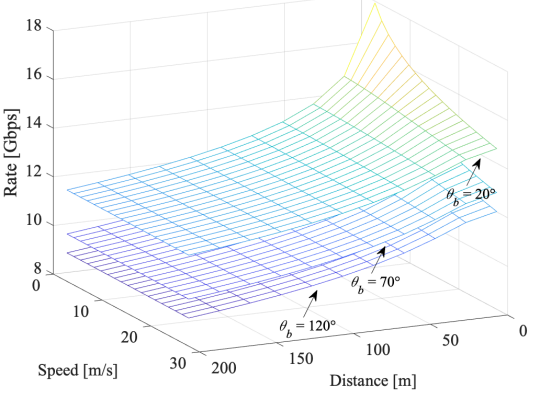

(c)

Fig. 8 - Evaluation of the instantaneous data rate [Gbps] vs. the beam width and the vehicle speed, in case of $(a) \theta_{b}=[0.1,10]^{\circ}$ and $(b) \theta_{b}=[30,120]^{\circ}$, both by considering different speeds i.e., $v_{1}=25, v_{2}=50$, and $v_{3}=75$ [m/s]. (c) Instantaneous data rate [Gbps] vs. vehicle speed and distances, in case of $\theta_{b}=[20,70,120]^{\circ}$.

Finally, in Fig. $8(c)$ we analyze the impact of the speed i.e., from 10 to $100 \mathrm{~km} / \mathrm{h}$, with respect to specific values of beam width i.e., $\theta_{b}=[20,70,120]^{\circ}$. The behavior follows the same trend as observed in Fig. $8(a)$ and $(b)$, where, as expected, for higher beam width the data rate performance is strongly reduced. Also, lower distances provide higher performance.

\subsection{Outage Probability}

The outage probability is a crucial metric for vehicular communication $[24,25]$, and can be defined as the instantaneous mutual information rate that falls below a certain threshold $\beta$, i.e.:

$$
\underset{\text { outage }}{\operatorname{Pr}}[S N R<\beta]=1-\underset{\text { success }}{\operatorname{Pr}},
$$

where $P r_{\text {success }}$ is the success probability that SNR is higher than $\beta$, and can be expressed as

$$
\underset{\text { success }}{\operatorname{Pr}}=\operatorname{Pr}[S N R>\beta]=\exp \left(-\beta d^{n} \frac{P_{\text {noise }}}{P_{t x}}\right),
$$

with $P_{t x}$ as the transmitting power.

Based on the outage probability, given a specific target rate as a Quality of Service (QoS) parameter, we can derive the throughput of success delivery with constrained outage probability $\epsilon$, as [24]:

$$
C=(1-\epsilon) B \log _{2}(1+S N R) .
$$

In order to evaluate the impact of the presence of the meta-surface, in Fig. 9, we consider a target rate of 1 Gbps and derive the corresponding outage probability as computed in Eq. (16). In order to better appreciate the effect of the meta-surface, we consider two different beam widths of $20^{\circ}$ and $90^{\circ}$. As expected, the lower is the beam width, the lower is the outage probability and the system has better performance in terms of capacity (see Fig. 10). The better performance is related to a higher precision of the system, that corresponds to an increased number of

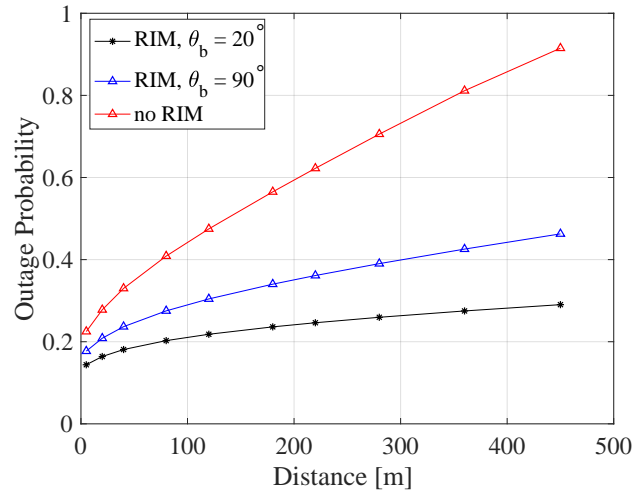

Fig. 9 - Outage Probability vs. the distance, in case of (i) a meta-surface with beam width $\theta_{b}=20^{\circ}$ and $\theta_{b}=90^{\circ}$, and (ii) without a metasurface.

meta-atoms needed in the metastructure for a high tuning of the phase. In practice, the higher is the number of unit cells of the full structure, the better is the control of the whole system, but also the control logic to drive the controllable meta-surface is more sophisticated/complicated, since it is demanding a higher precision and could make the system more vulnerable to misalignment errors.

\section{DISCUSSION}

In order to assess the performance of the system and to evaluate the potential impact of the RIM in a vehicular context, we have considered some assumptions to simplify the analysis and give some useful insights for future work. Firstly, a perfect alignment between the relay coated with the RIM and the receiver has been considered. This assumption cannot be ensured above all in a context characterised with high speed. In order to account for that, we need to introduce an error factor of the speed to account for the misalignment. A possible approach for accounting the misalignment has been proposed in [26]. We believe that this misalignment could be translated as a reduction of the received power and then in a decreasing of the SNR.

Another simplification introduced is the ideal configura- 


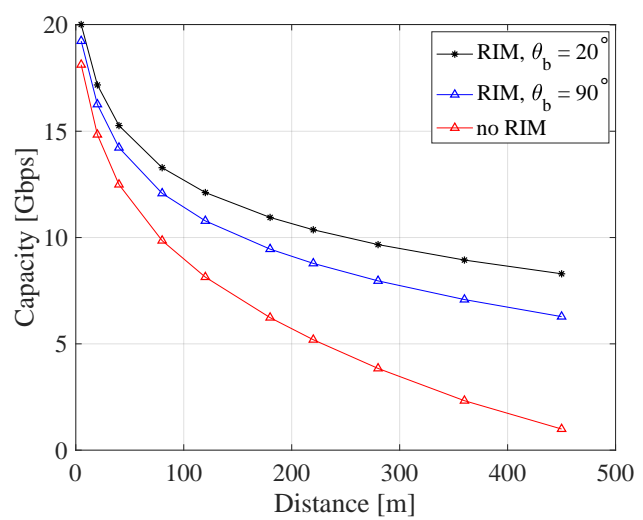

Fig. 10 - Capacity of the system with a rate set to $1 \mathrm{Gbps}$, in case of (i) a meta-surface with beam width $\theta_{b}=20^{\circ}$ and $\theta_{b}=90^{\circ}$, and (ii) without a meta-surface.

tion that is computed in real time and without error. Indeed, in order to control the RIM and make it as a tunable and intelligent meta-surface, we need to include an intelligent logic, potentially based on a machine learning (ML) approach to generate the correct configuration of the RIM when an external change impacts on the system (e.g., the vehicle moves far away from the RSU and a new beam needs to be calculated). ML approaches for systems based on RIM is a topic that is gaining more and more interest in recent times. Anyway, since we considered a mobile scenario where some crucial parameters can be easily predicted (e.g., the velocity of the vehicle and the direction), we trust that the computation of a new configuration can be calculated in real time and this should not impact too much on the ideal performance of the system.

\section{CONCLUSIONS}

In this paper, we presented a multifunctional reconfigurable meta-surface based on the radiation pattern modulation of the reflection coefficient. We designed a reconfigurable U-shaped unit cell using a pin diode via a hole, that allows to obtain a $180^{\circ}$-phase difference between $\mathrm{ON}$ and OFF states. The proposed RIM structure has been designed for multifunctional behavior such as coding and beam steering, considering a $10 \times 10$ meta-surface loaded with PIN diodes.

Simulation results have been carried out in order to validate the proposed RIM in a vehicular scenario, for data transmission via the V2X mode. The instantaneous data rate has been obtained, as a function of the beam width, the vehicle speed and the distance. As expected, the lower the beam width is, the higher the data rate is; additionally, the lower the vehicle speed is, the higher the data rate is. Same consideration occurs for the distance. In order to assess the effectiveness of the RIM integration in the vehicular communication system, we have derived the outage probability for a specific target QoS in terms of an instantaneous rate. We also have computed the capacity based on the outage probability and we have compared both the outage and the capacity for a system equipped with RIM with two different configurations in terms of beam width, and without RIM.

As a future piece of work, a factor that we have not considered in this context is the presence of other sources and interferences. We trust the integration of the RIM in the system can improve the reduction of the interference impact by properly addressing it. In order to account for this point, we plan to extend the analytical model with the interference factor and design RIM-based approaches that reduce the impact of the interference.

\section{ACKNOWLEDGMENT}

This work is partially supported by the Exploratory Action ETHICAM.

\section{REFERENCES}

[1] A. Nemati, Q. Wang, M. Hong, and J. Teng, "Tunable and reconfigurable meta-surfaces and metadevices," in Opto-Electronic Advances, 01, 180009, 2018.

[2] C. Liaskos, A. Tsioliaridou, S. Nie, A. Pitsillides, S. Ioannidis, and I. F. Akyildiz, "On the Network-layer Modeling and Configuration of Programmable Wireless Environments," in IEEE/ACM Transactions on Networking, vol. 27, no. 4, pp. 1696-1713, Aug. 2019.

[3] C. Liaskos, A. Tsioliaridou, A. Pitsillides, S. Ioannidis, and I. F. Akyildiz, "Using any Surface to Realize a New Paradigm for Wireless Communications," in Communications of the ACM, vol. 61 no. 11 , pp. 3033, October 2018.

[4] C. Liaskos, S. Nie, A. Tsioliaridou, A. Pitsillides, S. Ioannidis, and I. F. Akyildiz, "A New Wireless Communication Paradigm through Software-controlled Meta-surfaces," in IEEE Communications Magazine, vol. 56, no. 9, pp. 162-169, September 2018.

[5] L. La Spada, V. Loscrí, and A.M. Vegni, "Meta-Surface Structure Design and Channel Modelling for $\mathrm{THz}$ Band Communications," in Proc. of INFOCOM 2019 Workshop, 1st IEEE Workshop on Ultra-High Broadband Terahertz Communication for $5 \mathrm{G}$ and Beyond Networks (UBTCN 2019), April 29-May 2, Paris, France.

[6] A. Taibi, A. Durant, V. Loscrí, A.M. Vegni, and L. La Spada, "Controlling Light by Curvilinear MetaSurfaces," in Proc. of ACM Nanocom 2019, September 25-27, 2019, Dublin, Ireland.

[7] J. Dickmann et al., "Automotive radar the key technology for autonomous driving: From detection and ranging to environmental understanding," in Proc. of 2016 IEEE Radar Conference (RadarConf), Philadelphia, PA, 2016, pp. 1-6. 
[8] O. Schumann, J. Lombacher, M. Hahn, C. Wöhler and J. Dickmann, "Scene Understanding With Automotive Radar," in IEEE Transactions on Intelligent Vehicles, vol. 5, no. 2, pp. 188-203, June 2020, doi: 10.1109/TIV.2019.2955853.

[9] J. Wang, Y. Shao, Y. Ge, and R. Yu, "A Survey of Vehicle to Everything (V2X) Testing," in Sensors (Basel). 2019;19(2):334. Published 2019 Jan 15.

[10] J. Kim and A. F. Molisch, "Enabling Gigabit services for IEEE 802.11ad-capable high-speed train networks," in IEEE Radio and Wireless Symposium (RWS), pp. 145-147, Jan. 2013.

[11] H. Yang, X. Cao, F. Yang, J. Gao, S. Xu, M. Li, X. Chen, Y. Zhao, Y. Zheng, and S. Li, "A programmable metasurface with dynamic polarization, scattering and focusing control," in Scientific Reports, vol. 6, no. 35692, 2016.

[12] A. Khan, F. Ullah, Z. Kaleem, S. ur Rahman and Y. Z. Cho, "Sensor-based self-organized traffic control at intersections," in Proc. of 2017 International Conference on Information and Communication Technology Convergence (ICTC), Jeju, 2017, pp. 634-638, doi: 10.1109/ICTC.2017.8191056.

[13] N. Yu, P. Genevet, M. a. Kats, F. Aieta, J.-P. Tetienne, F. Capasso, and Z. Gaburro, "Light Propagation with Phase Discontinuities: Generalized Laws of Reflection and Refraction," in Science, vol. 334, no. October, pp. 333-337, 2011.

[14] S. Liu, T. Jun Cui, A. Noor, Z. Tao, H. Chi Zhang, G. Dong Bai, Y. Yang, and X. Yang Zhou, "Negative reflection and negative surface wave conversion from obliquely incident electromagnetic waves," in Light: Science and Applications, vol. 7, no. 5, pp. 18 008-18 011, 2018.

[15] T. Hamidreza, A. Cabellos-Aparicio, J. Georgiou, and S. Abadal, "Error Analysis of Programmable Metasurfaces for Beam Steering," in IEEE Journal on Emerging and Selected Topics in Circuits and Systems 10, no. 1 (2020): 62-74.

[16] H. Taghvaee et al., "Scalability Analysis of Programmable Meta-surfaces for Beam Steering," in IEEE Access, vol. 8, pp. 105320-105334, 2020, doi: 10.1109/ACCESS.2020.3000424.

[17] C. Rizza, V. Loscrí, and M. Parchin, "A MillimeterWave Reconfigurable Intelligent Meta-surface Design for Vehicular Networks Applications," in Proc. of 2020 IEEE Vehicular Technology Conference, Oct 2020, Victoria, Canada.

[18] L. Zhang, X. Qing Chen, S. Liu, Q. Zhang, J. Zhao, J. Yan Dai, G. Dong Bai, X. Wan, Q. Cheng, G. Castaldi, V. Galdi and T. Jun Cui, "Space-time-coding digital meta-surfaces," in Nature Communication, 9, 4334 (2018).

[19] L. Zhang, R. Y. Wu, G. D. Bai, H. T. Wu, Q. Ma, X. Q. Chen, T. J. Cui, "Transmission, Reflection, Integrated Multifunctional Coding Meta-surface for Full," in Space Controls of Electromagnetic Waves, in Adv. Funct. Mater. 2018, 28, 1802205.

[20] C. A. Balanis, Antenna Theory: Analysis and Design, 3rd ed., Wiley, Ed., 2005.

[21] E. Hosseininejad, K. Rouhi, M. Neshat, A. CabellosAparicio, S. Abadal, and E. Alarcon, "Digital Metasurface Based on Graphene: An Application to Beam Steering in Terahertz Plasmonic Antennas," in IEEE Transactions on Nanotechnology, vol. 18, no. 1, pp. 734-746, 2019.

[22] A. Makarf, K. M. Rabie, O. Kaiwartya, K. Adhikari, X. Li, M. Quiroz-Castellanos, and R. Kharel, "Reconfigurable intelligent surfaces-enabled vehicular networks: A physical layer security perspective," arXiv:2004.11288, 2020.

[23] B. Masini, M., Silva, M. Cristiano, and A. Balador, "The Use of Meta-Surfaces in Vehicular Networks," in Journal of Sensor and Actuator Networks, 2020, vol. 9, no 1, p. 15.

[24] X. Wu, S. Sun, Y. Li, Z. Tan, W. Huang, and X. Yao, "A Power Control Algorithm Based on Outage Probability Awareness in Vehicular Ad Hoc Networks," in Advances in Multimedia, vol. 2018, Article ID 8729645, 8 pages, 2018. https://doi.org/10.1155/2018/8729645

[25] B. E. Y. Belmekki, A. Hamza and B. Escrig, "On the Outage Probability of Vehicular Communications at Intersections Over Nakagami- $m$ Fading Channels," in Proc. of 2020 IEEE 91st Vehicular Technology Conference (VTC2020-Spring), Antwerp, Belgium, 2020, pp. 1-5, doi: 10.1109/VTC2020Spring48590.2020.9128618.

[26] V. Va, X. Zhang and R. W. Heath, "Beam Switching for Millimeter Wave Communication to Support High Speed Trains," in Proc. of 2015 IEEE 82nd Vehicular Technology Conference (VTC2015Fall), Boston, MA, 2015, pp. 1-5, doi: 10.1109/VTCFall.2015.7390855. 


\section{AUTHORS}

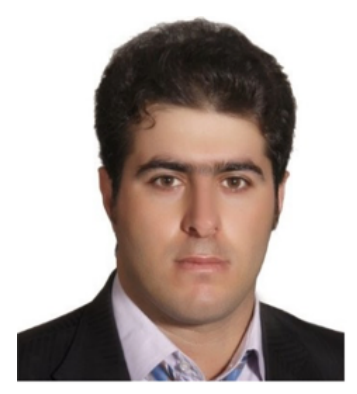

Mohammed Ojaroudi received a PhD degree at Shahid Beheshti University, Tehran, Iran, in 2014. He worked, as a visiting researcher at University of Tennessee, Knoxville, USA from 2013 to 2014. From Oct. 2014, he was a post-doctoral researcher at Middle East technical University (METU), Ankara, Turkey and from 2016 to 2017, he was an academic lecturer in Ankara University, Ankara, Turkey. From 2017-2020, he worked as a scientist researcher at XLIM research laboratory Limoges, France. From 2020 he has been working on a project in the frame of the reconfigurable intelligent meta-surface to develop short-range sensing for $\mathrm{THz}$ communication and imaging applications at Inria, Lille, France. From 2012, he has been a member of the IEEE-APS, IEEE-MTT, IEEE-AWPL, MAP-IET, reviewers' group. He is an author and coauthor of more than 150 journals and international conferences papers. His research interests include analysis and design of microstrip antennas, design and modeling of microwave structures, microwave imaging systems, and emerging electromagnetic technologies for $\mathrm{THz}$ communications.

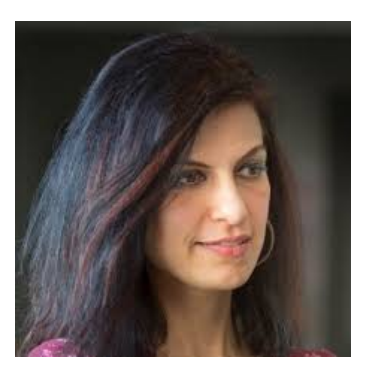

Valeria Loscrí is a permanent researcher of the FUN Team at Inria Lille-Nord Europe since Oct. 2013. From Dec. 2006 to Sept. 2013, she was Research Fellow in the TITAN Lab of the University of Calabria, Italy. She received her MSc and PhD degrees in Computer Science in 2003 and 2007, respectively, from the University of Calabria and her HDR (Habilitation a diriger des recherches) in 2018 from Université de Lille (France). Her research interests focus on emerging technologies for new communication paradigms such as Visible Light Communication and TeraHertz bandwidth and cooperation and coexistence of wireless heterogeneous devices. She has been involved in the activity of several European Projects (H2020 CyberSANE, FP7 EU project VITAL, the FP6 EU project
MASCOT, etc.), Italian and French projects. She is on the editorial board of IEEE COMST, Elsevier ComNet, JNCA, IEEE Trans. on Nanobioscience. Since 2019, she is Scientific International Delegate for Inria Lille-Nord Europe.

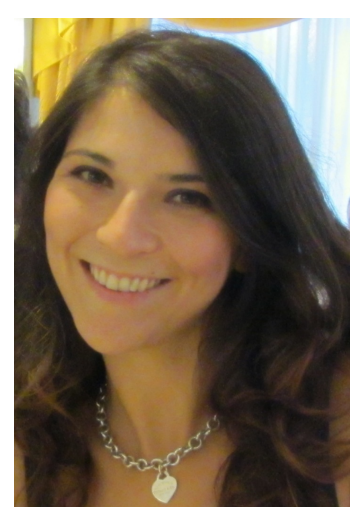

Anna Maria Vegni (Senior member, IEEE) is a tenuretrack Assistant Professor in the Department of Engineering at Roma Tre University (Italy), since March 2020. She received the Ph.D. degree in Biomedical Engineering, Electromagnetics and Telecommunications from the Department of Applied Electronics, Roma Tre University, in March 2010. She received the 1st and 2nd level Laurea Degree cum laude in Electronics Engineering at Roma Tre University, in July 2004, and 2006, respectively. In 2009, she was a visiting researcher in the Multimedia Communication Laboratory, directed by Prof. Thomas D.C. Little, at the Department of Electrical and Computer Engineering, Boston University, Boston, MA. Her research activity focused on vehicular networking supported by heterogeneous wireless networks and optical wireless communications. She is a member of ACM and an IEEE Senior Member. In March 2018, she got the Italian Habilitation (Abilitazione Scientifica Nazionale) for Associate Professorship in Telecommunication Engineering. She is involved in the organization of several IEEE and ACM international conferences and is a member of the editorial board of IEEE Communications Magazine, Ad Hoc Networks, Journal of Networks and Computer Applications, Nanocomnet Elsevier journals, IEEE JCN, ITU J-FET and ETT Wiley journal. 